\title{
RIKEN WT Zebrafish
}

National Cancer Institute

\section{Source}

National Cancer Institute. RIKEN WT Zebrafish. NCI Thesaurus. Code C79979.

A wild-type zebrafish line that is currently available from the National BioResource

Project, Zebrafish, NRBP/Brain Science Institute, RIKEN. 